\title{
Revealing Tensor Monopoles through Quantum-Metric Measurements
}

\author{
Giandomenico Palumbo and Nathan Goldman \\ Center for Nonlinear Phenomena and Complex Systems, Université Libre de Bruxelles, \\ CP 231, Campus Plaine, B-1050 Brussels, Belgium
}

(Received 9 May 2018; published 24 October 2018)

\begin{abstract}
Monopoles are intriguing topological objects, which play a central role in gauge theories and topological states of matter. While conventional monopoles are found in odd-dimensional flat spaces, such as the Dirac monopole in three dimensions and the non-Abelian Yang monopole in five dimensions, more exotic objects were predicted to exist in even dimensions. This is the case of "tensor monopoles," which are associated with tensor (Kalb-Ramond) gauge fields, and which can be defined in four-dimensional flat spaces. In this work, we investigate the possibility of creating and measuring such a tensor monopole in condensed-matter physics by introducing a realistic three-band model defined over a four-dimensional parameter space. Our probing method is based on the observation that the topological charge of this tensor monopole, which we relate to a generalized Berry curvature, can be directly extracted from the quantum metric. We propose a realistic three-level atomic system, where tensor monopoles could be generated and revealed through quantum-metric measurements.
\end{abstract}

DOI: 10.1103/PhysRevLett.121.170401

Introduction.-Magnetic monopoles were originally introduced by Dirac in 1931 [1], in view of proving the quantization of the electric charge in quantum electrodynamics. While the Dirac monopole has played an important role in high-energy physics, in particular due to its topological nature [2,3], an experimental confirmation of its existence is still lacking. Since Dirac's original work, a zoo of monopoles have been identified in the context of gauge theory. Prominent examples are the 't Hooft-Polyakov monopole [4,5] found in Yang-Mills theory coupled to a Higgs field, and the non-Abelian monopole introduced by Yang [6], which can exist in five dimensions (5D). Importantly, all these monopoles carry a quantized "magnetic" charge, which has a topological origin. Specifically, the monopole charge can be related to a topological invariant [2], which is given by the real-space integral of the curvature (or field strength tensor) associated with the monopole's gauge field [7]. This topological invariant corresponds to the "first Chern number" in the case of three-dimensional (3D) monopoles [2], while the "second Chern number" appears as the relevant invariant in 5D [6].

The monopoles mentioned above are all associated with vector gauge fields, an example of which is the well-known electromagnetic gauge potential [2]. However, it was suggested that tensor gauge fields could also be defined and that these more exotic gauge structures could also give rise to monopoles [8-10]. In this distinct class of monopoles, the simplest representative is the so-called "tensor monopole": an Abelian monopole defined in a fourdimensional (4D) space and whose magnetic charge is given by the integral of the curvature associated with a tensor (Kalb-Ramond) gauge field [11], which represents a direct generalization of the electromagnetic potential [12]. It also plays an important role in string theory, where currents naturally couple to a tensor gauge field [13-15].

The search for monopoles is of fundamental relevance in high-energy physics, and their detection still constitutes a severe challenge. So far, none of these topological objects has been identified, and there is no concrete evidence that these could be accessed in current experiments. However, monopoles also naturally appear in condensed-matter physics, where various forms of effective gauge potentials can emerge. A prominent example is the so-called Berry connection, which plays the role of a gauge potential in the parameter space of a quantum system, and which is responsible for the geometric (Berry) phase $[7,16]$. Importantly, the monopoles associated with the Berry connection were shown to be deeply connected to the existence of topological states of matter [17,18]; see also Ref. [19] on monopoles in spin ice. For instance, the topological invariant associated with the quantum Hall effects $[16,18]$ and the recently discovered Weyl semimetals $[20,21]$, the so-called Chern number, can be simply attributed to fictitious Dirac monopoles defined in momentum space. Moreover, artificial Dirac and Yang monopoles have been implemented in ultracold atoms [22,23], where the high control over the physical parameters allows for tunable synthetic gauge potentials $[24,25]$.

The goal of this work is twofold. First, we demonstrate how monopoles associated with the Berry connection of a quantum system can be directly extracted from the quantum-metric tensor (or Fubini-Study or Bures metric) [26], following an approach initially developed in high-energy physics for treating real-space monopoles $[8,27]$. This 
result is particularly useful for systems where the Berry curvature cannot be measured or calculated explicitly, as we further discuss below. We remind the reader that the quantum metric is related to several physical observables [28-32] and that it could be directly measured in cold atoms [33].

Second, we introduce a minimal three-level model realizing a tensor monopole in a $4 \mathrm{D}$ parameter space. This model, which is inspired by the recent proposal [34] for realizing spin-1 monopoles, could be realized in ultracold gases by coupling three internal states of an atom. In particular, it generalizes in a nontrivial way the recent experimental setting that realized non-Abelian Yang monopoles in cold atoms [23]: As stated above, the tensor monopole resulting from our model is defined in a $4 \mathrm{D}$ parameter space, and its topological charge is related to the existence of a generalized Berry curvature associated with a tensor gauge field. In this Letter, we discuss how this exotic topological object could be extracted from quantum-metric measurements [33]. Moreover, our model exhibits an intriguing topological state that gives rise to a nontrivial generalization of Weyl semimetals in 4D, similar to that recently proposed in Ref. [35]. This work represents a first step towards the analysis and implementation of novel classes of monopoles and higher-dimensional topological states of matter in quantum engineered systems [36].

Monopoles and the quantum metric.-We start by recalling notions related to the Dirac monopole in the context of electromagnetism. Defining the electromagnetic potential $A_{\mu}$, the charge of a monopole located in $\mathbb{R}^{3}$ is obtained by integrating the Faraday tensor $\mathcal{F}_{\mu \nu}=\partial_{\mu} A_{\nu}-\partial_{\nu} A_{\mu}$ over a sphere $S^{2}$ that surrounds it; see Fig. 1(a). This nonvanishing
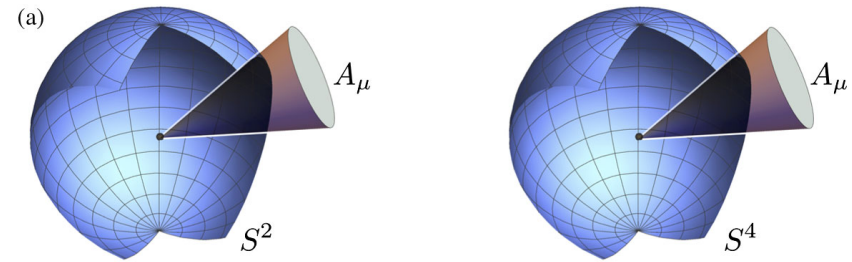

(b)

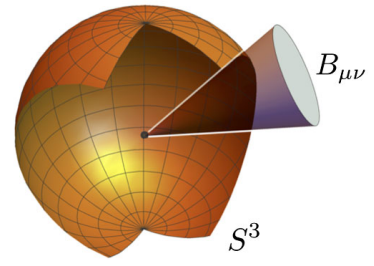

FIG. 1. (a) Pictorial representations of a Dirac monopole in 3D (left) and of a Yang monopole in 5D (right). Both are defined as pointlike objects, which are sources of a vector gauge field $A_{\mu}$. The total flux associated with $A_{\mu}$ through the surrounding spheres ( $S^{2}$ and $S^{4}$, respectively) is quantized in terms of the first (resp., second) topological Chern number. (b) A tensor monopole is a pointlike source of a tensor (Kalb-Ramond) gauge field $B_{\mu \nu}$, which can exist in a 4D space. The corresponding flux that comes out of the surrounding sphere $S^{3}$ is also quantized [Eq. (10)]. flux identifies the charge $(1 / 2 \pi) \int_{S^{2}} \mathcal{F}=\nu^{1}$, which is quantized in terms of the topological Chern number $\nu^{1}$; here we introduced the 2-form $\mathcal{F}=d A=(1 / 2) \mathcal{F}_{\mu \nu} d x^{\mu} \wedge d x^{\nu}$, the wedge $(\wedge)$ product, and the exterior $(d)$ derivative [7]. Since the magnetic field emanating from a monopole is purely radial, the calculation of the total flux through $S^{2}$ essentially reduces to calculating the sphere's surface. This suggests an interesting relation between the Faraday tensor associated with a monopole and the determinant of the metric tensor $g_{\mu \nu}$, which is defined on a sphere surrounding it $[8,27]$ :

$$
\mathcal{F}_{\mu \nu}=\epsilon_{\mu \nu} k \sqrt{\operatorname{det} g_{\mu \nu}},
$$

where $k$ is a suitable normalization constant, and $\epsilon_{\mu \nu}$ is the Levi-Cività symbol. Note that the expression (1) for the Faraday tensor is still gauge invariant and antisymmetric. Remarkably, it was demonstrated that such a relation between the Faraday tensor and a sphere's metric is not specific to the Dirac monopole: It can be systematically generalized to any pointlike or extended monopole in any spatial dimension $[8,27]$. In particular, this approach is particularly useful to identify tensor monopoles [8], as will be illustrated below.

We first describe how this approach applies to fictitious Dirac monopoles, as defined in the parameter space of a quantum system. Consider an eigenstate $|u(\boldsymbol{q})\rangle$ of a quantum system defined over some $3 \mathrm{D}$ parameter space spanned by $\boldsymbol{q}$ (e.g., a state in a given Bloch band with quasimomentum $\boldsymbol{q}$ ). The geometric properties of this state are captured by the quantum geometric tensor, which can be split into real and imaginary parts [26,29,30]: $\chi_{\mu \nu}=g_{\mu \nu}+(i / 2) \mathcal{F}_{\mu \nu}$, where $\mathcal{F}_{\mu \nu}=\partial_{\mu} A_{\nu}-\partial_{\nu} A_{\mu}$ is the Berry curvature, $A_{\mu}=i\left\langle u \mid \partial_{\mu} u\right\rangle$ is the (Abelian) Berry connection, and where

$$
\begin{aligned}
g_{\mu \nu}= & \frac{1}{2}\left(\left\langle\partial_{\mu} u \mid \partial_{\nu} u\right\rangle+\left\langle\partial_{\nu} u \mid \partial_{\mu} u\right\rangle\right. \\
& \left.-\left\langle\partial_{\mu} u \mid u\right\rangle\left\langle u \mid \partial_{\nu} u\right\rangle-\left\langle\partial_{\nu} u \mid u\right\rangle\left\langle u \mid \partial_{\mu} u\right\rangle\right),
\end{aligned}
$$

is the quantum-metric tensor $[26,29,30]$. Here, all derivatives are taken with respect to the parameters, i.e., $\partial_{\mu}=\partial_{q_{\mu}}$. While the Berry curvature $\mathcal{F}$ is associated with the geometric (Berry) phase [16] and can be viewed as a Faraday tensor in $\boldsymbol{q}$ space [16,36], the quantum metric $g$ measures the (infinitesimal) distance between two nearby quantum states in $\boldsymbol{q}$ space; see Refs. [28-33,37-39] for physical manifestations of the quantum metric.

In analogy with electromagnetism, a finite Chern number $\nu^{1}=(1 / 2 \pi) \int_{S^{2}} \mathcal{F}$ signals the presence of a fictitious monopole in $\boldsymbol{q}$ space [21] located inside some sphere $S^{2}$. Such objects appear in the context of Weyl semimetals, where the vector $\boldsymbol{q}$ represents the quasimomentum of a lattice system [21]. Inspired by Eq. (1), we now propose that such monopoles can also be detected through the 
determinant of a metric: the quantum-metric tensor in Eq. (2). To illustrate this approach, we consider a minimal model realizing a monopole in parameter space: the Weyl Hamiltonian [21]

$$
\hat{H}_{3 \mathrm{D}}=q_{x} \sigma^{x}+q_{y} \sigma^{y}+q_{z} \sigma^{z},
$$

where $\boldsymbol{q}=\left(q_{x}, q_{y}, q_{z}\right)$ denotes the momentum and where $\sigma^{x, y, z}$ are the Pauli matrices. The corresponding energy spectrum supports a Weyl cone $E= \pm \sqrt{q_{x}^{2}+q_{y}^{2}+q_{z}^{2}}$, and the Berry curvature associated with the low-energy eigenvector $\left|u_{-}(\boldsymbol{q})\right\rangle$ reads

$$
\mathcal{F}=\mathcal{F}_{\mu \nu} d q_{\mu} \wedge d q_{\nu}, \quad \mathcal{F}_{\mu \nu}=\epsilon_{\mu \nu \lambda} \frac{q_{\lambda}}{2\left(q_{x}^{2}+q_{y}^{2}+q_{z}^{2}\right)^{3 / 2}} .
$$

This corresponds to the Faraday tensor associated with a fictitious Dirac monopole at $\boldsymbol{q}=0$, as can be verified by calculating the corresponding charge; see Eq. (6) below. Inspired by Eq. (1) and the approach of Refs. [8,27], we now show that this topological charge can be obtained from the determinant of the quantum-metric tensor. Introducing the spherical coordinates $q_{x}=r \sin \theta \cos \phi$, $q_{y}=r \sin \theta \sin \phi, q_{z}=r \cos \theta$, the components of the quantum-metric tensor (2) associated with the eigenvector $\left|u_{-}(\boldsymbol{q})\right\rangle$ read [33]

$$
g_{\theta \theta}=1 / 4, \quad g_{\phi \phi}=\sin ^{2} \theta / 4, \quad g_{\theta \phi}=0 .
$$

This corresponds to the metric of a sphere $S^{2}$ of fixed radius $r=1 / 2$, which surrounds the Weyl node (i.e., the monopole) in $\boldsymbol{q}$ space. Hence, the topological charge $Q$ of the Dirac monopole is encoded in the quantum metric through the relation

$$
Q=\frac{1}{2 \pi} \int_{S^{2}} \mathcal{F}=\frac{1}{2 \pi} \iint(2 \sqrt{g}) d \theta d \phi=1,
$$

where $g=\operatorname{det} g_{a b}$ is the determinant of the $2 \times 2$ metric tensor in Eq. (5), with $a, b=\{\theta, \phi\}$. Going back to Cartesian coordinates, we find a direct relation between the components of the Berry curvature in Eq. (4) and the quantum metric [Eq. (2)] associated with $\left|u_{-}(\boldsymbol{q})\right\rangle$,

$$
\mathcal{F}_{\mu \nu}=\epsilon_{\mu \nu}\left(2 \sqrt{\operatorname{det} g_{\bar{\mu} \bar{\nu}}}\right),
$$

where $g_{\bar{\mu} \bar{\nu}}$ is the $2 \times 2$ quantum-metric tensor defined in the proper 2D subspace (e.g., $q_{\bar{\mu}}, q_{\bar{\nu}}=\left\{q_{x}, q_{y}\right\}$ for the calculation of $\mathcal{F}_{x y}$ ). Equation (7) is the direct analogue of Eq. (1) when applied to the Berry curvature, and it indicates how quantum-metric measurements [30,33] could directly reveal Dirac monopoles in $\boldsymbol{q}$ space. It is the scope of the

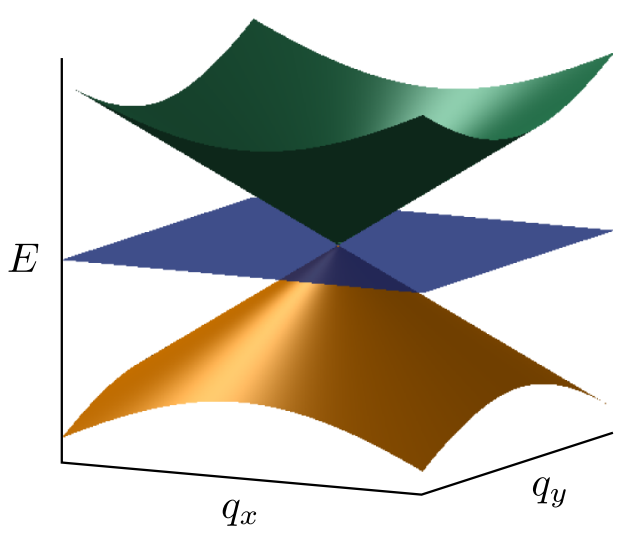

FIG. 2. Energy dispersion of the three-level Hamiltonian $H_{4 \mathrm{D}}$ in Eq. (11), at $q_{z}=q_{w}=0$. One eigenvalue of the Hamiltonian is always zero, and there appears a degenerate triple point at $\boldsymbol{q}_{D}=(0,0,0,0)$, where the tensor monopole lies. This threeband configuration, with a single degenerate triple point, offers a minimal setting realizing an Abelian tensor monopole [40]. This spectrum is also reminiscent of that found in 3D spin-1 Weyl semimetals [41] and spin-1 monopoles [34].

next paragraph to apply such an approach to a 4D Weyl-like Hamiltonian supporting a tensor monopole [8].

Tensor monopoles in $4 D$ parameter space.-As recalled in the previous paragraph, Weyl nodes are sources of Dirac monopoles in 3D parameter space [21]. This phenomenon can be generalized to Dirac-like nodes exhibiting a triplepoint crossing, which lead to so-called spin-1 monopoles [34,41]. Regarding higher dimensions, Dirac nodes appearing in 5D parameter space were shown to be associated with non-Abelian Yang monopoles [23,42,43]; see also Ref. [44] on extended monopoles in 5D. Importantly, all these monopoles defined in 3D and 5D spaces are described by conventional (vector) Berry connections [16], i.e., vector gauge fields. In this paragraph, we explore monopoles defined in 4D spaces, which are captured by tensor Berry connections [45,46,51], i.e., tensor gauge fields [8].

Tensor gauge fields have been mainly analyzed in the context of high-energy physics [8,9,11-15], with some applications in topological states of matter [52-61]. The idea consists of introducing an Abelian antisymmetric tensor field $B_{\mu \nu}$ called the Kalb-Ramond field, which naturally generalizes the usual electromagnetic potential $A_{\mu}[11,12]$. It transforms under a $U(1)$ gauge transformation as follows:

$$
B_{\mu \nu} \rightarrow B_{\mu \nu}+\partial_{\mu} \xi_{\nu}-\partial_{\nu} \xi_{\mu}
$$

where $\xi_{\mu}$ is a vector that contains the redundant gauge degree of freedom of the field $B_{\mu \nu}$. The corresponding (3-form) curvature tensor $\mathcal{H}=d B$ has components

$$
\mathcal{H}_{\mu \nu \lambda}=\partial_{\mu} B_{\nu \lambda}+\partial_{\nu} B_{\lambda \mu}+\partial_{\lambda} B_{\mu \nu}
$$


It is gauge invariant and antisymmetric, in analogy with the Faraday tensor. As shown in Refs. [8,9], $B_{\mu \nu}$ gives rise to a novel type of monopole in 4D space, which is Abelian and pointlike: This tensor monopole generalizes the 3D Dirac monopole to 4D. Specifically, if one surrounds the tensor monopole with a three-dimensional sphere $S^{3}$ [see Fig. 1(b)], one can derive a topological charge associated with the (3-form) curvature $\mathcal{H}_{\mu \nu \lambda}$,

$$
Q_{T}=\frac{1}{2 \pi^{2}} \int_{S^{3}} d x^{\mu} \wedge d x^{\nu} \wedge d x^{\lambda} \mathcal{H}_{\mu \nu \lambda} .
$$

This is a topological invariant known as the DixmierDouady invariant $[35,62,63]$, a generalization of the betterknown Chern number [7]; this invariant is associated with the third homotopy group $\pi_{3}\left(S^{3}\right)=\mathbb{Z}$ and characterizes a $U(1)$ "bundle gerbe" [64,65]. Importantly, the result in Eq. (1) can be directly generalized to tensor monopoles [8], which indicates that the topological charge in Eq. (10) can be calculated through the determinant of the metric tensor defined on $S^{3}$.

The goal of this paragraph is to show how the parameterspace (Berry-type) analogue of the curvature $\mathcal{H}_{\mu \nu \lambda}$ [Eq. (9)] as well as its topological charge [Eq. (10)] can be determined through the quantum-metric tensor of a quantum system. To do so, we consider a minimal Weyl-type Hamiltonian defined in a 4D parameter space [40] spanned by $\boldsymbol{q}=\left(q_{x}, q_{y}, q_{z}, q_{w}\right)$,

$$
\begin{aligned}
\hat{H}_{4 \mathrm{D}} & =q_{x} \lambda_{1}+q_{y} \lambda_{2}+q_{z} \lambda_{6}+q_{w} \lambda_{7}^{*}, \\
& =\left(\begin{array}{ccc}
0 & q_{x}-i q_{y} & 0 \\
q_{x}+i q_{y} & 0 & q_{z}+i q_{w} \\
0 & q_{z}-i q_{w} & 0
\end{array}\right),
\end{aligned}
$$

where the $\lambda$ matrices are $3 \times 3$ Gell-Mann matrices [66]

The corresponding spectrum is given by

$$
E_{0}=0, \quad E_{ \pm}= \pm \sqrt{q_{x}^{2}+q_{y}^{2}+q_{z}^{2}+q_{w}^{2}},
$$

where we recognize the presence of a triple-degenerate Dirac-like point at $\boldsymbol{q}_{\mathrm{D}}=(0,0,0,0)$; see Fig. 2 . We now demonstrate that this 4D Dirac-like node is a source of a tensor monopole based on the approach developed in the previous paragraph: Inspired by Ref. [8] and Eqs. (7) and (9), we write the generalized Berry curvature tensor as

$$
\mathcal{H}_{\mu \nu \lambda}=\epsilon_{\mu \nu \lambda}\left(4 \sqrt{\operatorname{det} g_{\bar{\mu} \bar{\nu}}}\right),
$$

where $g_{\bar{\mu} \bar{\nu}}$ is the $3 \times 3$ quantum-metric tensor defined in the proper 3D subspace (e.g., $q_{\bar{\mu}}, q_{\bar{\nu}}=\left\{q_{x}, q_{y}, q_{z}\right\}$ for the calculation of $\left.\mathcal{H}_{x y z}\right)$. This quantum metric can be directly evaluated using Eq. (2), and the expression for the low-energy eigenstate of $\hat{H}_{4 \mathrm{D}}$ in Eq. (11): $\left|u_{-}\right\rangle=(1 / \sqrt{2})\left(v_{1},-1, v_{2}\right)^{\top}$,

$$
v_{1}=\frac{q_{x}-i q_{y}}{|E|}, \quad v_{2}=\frac{q_{z}-i q_{w}}{|E|} .
$$

Similar to the case of the 3D Weyl Hamiltonian [Eq. (5)], we find that the corresponding quantum metric identifies a three-sphere $S^{3}$ with fixed radius surrounding the Dirac-like node. Furthermore, when combined with the ansatz in Eq. (13), we obtain

$$
\mathcal{H}_{\mu \nu \lambda}=\epsilon_{\mu \nu \lambda \gamma} \frac{q_{\gamma}}{\left(q_{x}^{2}+q_{y}^{2}+q_{z}^{2}+q_{w}^{2}\right)^{2}},
$$

which indeed coincides with the curvature of a tensor monopole in 4D [8]. Introducing the hyperspherical coordinates $\left(r, \theta_{1}, \theta_{2}, \varphi\right)$ (see Ref. [67]), we explicitly calculate the topological charge $Q_{T}$ associated with $\mathcal{H}_{\mu \nu \lambda}$,

$$
\begin{aligned}
Q_{T} & =\frac{1}{2 \pi^{2}} \int_{S^{3}} d q^{\mu} \wedge d q^{\nu} \wedge d q^{\lambda} \mathcal{H}_{\mu \nu \lambda} \\
& =\frac{1}{2 \pi^{2}} \int_{0}^{\pi} d \theta_{1} \int_{0}^{\pi} d \theta_{2} \int_{0}^{2 \pi} d \varphi \sin ^{2} \theta_{1} \sin \theta_{2}=1 .
\end{aligned}
$$

One verifies that the charge $Q_{T}$ is immune to smooth deformations of the Hamiltonian that preserve the Diraclike node, in agreement with its topological nature.

We point out that the topological charge in Eq. (16) was identified through the quantum metric only, using the relation in Eq. (13). An alternative approach consists of determining the 2-form Berry connection $B_{\mu \nu}$, which is related to the curvature through $\mathcal{H}=d B$. As shown in Refs. [46,51], a proper 2-form Berry connection associated with the state in Eq. (14) can be expressed as

$$
B_{\mu \nu}=\frac{i}{3} \sum_{j, k, l=1}^{3} \epsilon^{j k l} \phi_{j} \partial_{\mu} \phi_{k} \partial_{\nu} \phi_{l},
$$

where $\phi_{1}=-i \log v_{2}$ and $\phi_{2}=\phi_{3}^{*}=v_{1}^{*}$. One verifies that the corresponding curvature $\mathcal{H}_{\mu \nu \lambda}=\partial_{\mu} B_{\nu \lambda}+\partial_{\nu} B_{\lambda \mu}+$ $\partial_{\lambda} B_{\mu \nu}$ indeed reproduces the result in Eq. (15).

If we identify the parameters $q$ with the quasimomenta of a suitable 4D lattice model, Eq. (11) can be seen as the linearized Hamiltonian of a generalized Weyl semimetal defined in 4D, similar to that proposed in Ref. [35]. Moreover, by taking a slice of our 4D model, at some fixed $q_{w}=$ const, we obtain a three-dimensional gapped phase, which describes a topological insulator in the chiral class AIII; indeed, the Hamiltonian $\hat{H}_{4 \mathrm{D}}$ preserves chiral symmetry according to

$$
U \hat{H}_{4 \mathrm{D}}(\mathbf{q}) U^{-1}=-\hat{H}_{4 \mathrm{D}}(\mathbf{q}), \quad U=\left(\begin{array}{ccc}
1 & 0 & 0 \\
0 & -1 & 0 \\
0 & 0 & 1
\end{array}\right) \text {. }
$$

Such AIII topological phases were recently explored in a 3D model [68]. In principle, 4D lattice models leading to 
the Hamiltonian in Eq. (11) could be realized in optical lattices, e.g., using synthetic dimensions $[69,70]$.

Ultracold-atoms implementation.-In this paragraph, we present a possible physical realization of a tensor monopole through the manipulation of three atomic levels. A natural choice would be three sublevels within the hyperfine ground states of ${ }^{87} \mathrm{Rb}$ atoms coupled by two (rf or microwave) driving fields $[23,34]$. The corresponding three-level Hamiltonian can be written in the general form

$$
\hat{H}_{\exp }=\left(\begin{array}{ccc}
-\delta_{12} & \omega_{12} e^{-i \phi_{12}} & 0 \\
\omega_{12} e^{i \phi_{12}} & 0 & \omega_{23} e^{i \phi_{23}} \\
0 & \omega_{23} e^{-i \phi_{23}} & \delta_{23}
\end{array}\right),
$$

where $\omega_{i j}$ and $\phi_{i j}$ are the Rabi amplitudes and phases of the coupling fields [24,25], respectively, and where $\delta_{i j}$ capture the detuning from resonances. In contrast with previous proposals [34], we now neglect these detuning effects $\left(\delta_{i j}=0\right)$, which we assume to be small compared to the Rabi amplitudes. In this regime, the Hamiltonian $\hat{H}_{\text {exp }}$ depends on four independent parameters only, and it can therefore be mapped onto the Hamiltonian in Eq. (11) through the following identifications:

$$
\omega_{12} e^{i \phi_{12}}=q_{x}+i q_{y}, \quad \omega_{23} e^{i \phi_{23}}=q_{z}+i q_{w} .
$$

Hence, this minimal platform supports a (fictitious) tensor monopole in the parameter space spanned by $\left\{\omega_{12}, \phi_{12}, \omega_{23}, \phi_{23}\right\}$, and it is characterized by a nonzero topological charge $Q_{T}$; see Eq. (16). As shown in the previous paragraph, this topological invariant can be directly obtained from the quantum metric associated with the Hamiltonian's eigenstates. Following the protocol of Ref. [33], the components of the quantum metric could be individually obtained by initially preparing the system in the low-energy eigenstate of the Hamiltonian (19) and then monitoring the excitation rate upon modulating the system parameters in time [46]; see Ref. [71] for a recent measurement and Ref. [30] for other possible probes of the metric. This would represent a direct method to reveal, for the first time, the existence of tensor monopoles through the identification of their monopole charge via quantummetric measurements.

We are grateful to $\mathrm{M}$. Di Liberto for a careful reading of a preliminary version of the manuscript and to M. Aidelsburger, B. Mera, and T. Ozawa for helpful discussions. This work was supported by the Fonds De La Recherche Scientifique (FRS-FNRS) (Belgium) and the ERC Starting Grant TopoCold.

[1] P. A. M. Dirac, Proc. R. Soc. A 133, 60 (1931).

[2] T. T. Wu and C. N. Yang, Phys. Rev. D 12, 3845 (1975).

[3] T. T. Wu and C. N. Yang, Nucl. Phys. B107, 365 (1976).
[4] A. M. Polyakov, JETP Lett. 20, 194 (1974).

[5] G. 't Hooft, Nucl. Phys. B79, 276 (1974).

[6] C. N. Yang, J. Math. Phys. (N.Y.) 19, 320 (1978).

[7] M. Nakahara, Geometry, Topology, and Physics (Institute of Physics Publishing, Bristol, 2003).

[8] R. I. Nepomechie, Phys. Rev. D 31, 1921 (1985).

[9] C. Teitelboim, Phys. Lett. 167B, 69 (1986).

[10] P. Orland, Nucl. Phys. B205, 107 (1982).

[11] M. Kalb and P. Ramond, Phys. Rev. D 9, 2273 (1974).

[12] M. Henneaux and C. Teitelboim, Found. Phys. 16, 593 (1986).

[13] T. Banks and N. Seiberg, Phys. Rev. D 83, 084019 (2011).

[14] N. E. Mavromatos and S. Sarkar, Phys. Rev. D 95, 104025 (2017).

[15] M. Montero, A. M. Uranga, and I. Valenzuela, J. High Energy Phys. 07 (2017) 123.

[16] D. Xiao, M.-C. Chang, and Q. Niu, Rev. Mod. Phys. 82, 1959 (2010).

[17] E. Fradkin, Field Theories of Condensed Matter Physics (Cambridge University Press, Cambridge, England, 2013).

[18] X.-L. Qi and S.-C. Zhang, Rev. Mod. Phys. 83, 1057 (2011).

[19] C. Castelnovo, R. Moessner, and S. L. Sondhi, Nature (London) 451, 42 (2008).

[20] X. Wan, A. M. Turner, A. Vishwanath, and S. Y. Savrasov, Phys. Rev. B 83, 205101 (2011).

[21] N. P. Armitage, E. J. Mele, and A. Vishwanath, Rev. Mod. Phys. 90, 015001 (2018).

[22] M. W. Ray, E. Ruokokoski, S. Kandel, M. Mottonen, and D. S. Hall, Nature (London) 505, 657 (2014).

[23] S. Sugawa, F. Salces-Carcoba, A. R. Perry, Y. Yue, and I. B. Spielman, Science 360, 1429 (2018).

[24] J. Dalibard, F. Gerbier, G. Juzeliunas, and P. Ohberg, Rev. Mod. Phys. 83, 1523 (2011).

[25] N. Goldman, G. Juzeliunas, P. Ohberg, and I. B. Spielman, Rep. Prog. Phys. 77, 126401 (2014).

[26] G. P. Provost and G. Vallee, Commun. Math. Phys. 76, 289 (1980).

[27] P. G. O. Freund and M. A. Rubin, Phys. Lett. 97B, 233 (1980).

[28] R. Resta, Eur. Phys. J. B 79, 121 (2011).

[29] R. Roy, Phys. Rev. B 90, 165139 (2014).

[30] M. Kolodrubetz, V. Gritsev, and A. Polkovnikov, Phys. Rev. B 88, 064304 (2013).

[31] T. Ozawa, Phys. Rev. B 97, 041108(R) (2018).

[32] G. Palumbo, Eur. Phys. J. Plus 133, 23 (2018).

[33] T. Ozawa and N. Goldman, Phys. Rev. B 97, 201117(R) (2018).

[34] H. Hu and C. Zhang, Phys. Rev. A 98, 013627 (2018).

[35] V. Mathai and G. C. Thiang, Commun. Math. Phys. 355, 561 (2017).

[36] M. Aidelsburger, S. Nascimbene, and N. Goldman, arXiv:1710.00851.

[37] A. Raoux, F. Piechon, J.-N. Fuchs, and G. Montambaux, Phys. Rev. B 91, 085120 (2015).

[38] F. Piechon, A. Raoux, J.-N. Fuchs, and G. Montambaux, Phys. Rev. B 94, 134423 (2016).

[39] Y. Gao, S. A. Yang, and Q. Niu, Phys. Rev. B 91, 214405 (2015). 
[40] Tensor monopoles can exist in parameter spaces of dimension $D \geq 4$; see Ref. [8]. A three-band model defined in 4D, which exhibits a single degenerate triple point, constitutes a minimal setting for the realization of an Abelian tensor monopole.

[41] I. C. Fulga, L. Fallani, and M. Burrello, Phys. Rev. B 97, 121402(R) (2018).

[42] R. Shankar and H. Mathur, Phys. Rev. Lett. 73, 1565 (1994).

[43] J. E. Avron, L. Sadun, J. Segert, and B. Simon, Phys. Rev. Lett. 61, 1329 (1988).

[44] B. Lian and S.-C. Zhang, Phys. Rev. B 94, 041105(R) (2016).

[45] The concept of a tensor Berry connection was also suggested in Ref. [35] but without providing any explicit expression for the corresponding curvature.

[46] See Supplemental Material at http://link.aps.org/ supplemental/10.1103/PhysRevLett.121.170401 for more details about tensor Berry connections and possible detection schemes, which includes Refs. [47-50].

[47] G. Dvali, arXiv:hep-th/0507215.

[48] E. I. Guendelman, E. Nissimov, and S. Pacheva, Phys. Lett. B 360, 57 (1995).

[49] N. Fläschner, B. S. Rem, M. Tarnowski, D. Vogel, D. S. Lühmann, K. Sengstock, and C. Weitenberg, Science 352, 1091 (2016).

[50] R. Bianchetti, S. Filipp, M. Baur, J. M. Fink, C. Lang, L. Steffen, M. Boissonneault, A. Blais, and A. Wallraff, Phys. Rev. Lett. 105, 223601 (2010).

[51] G. Palumbo and N. Goldman (to be published).

[52] T. H. Hansson, V. Oganesyan, and S. L. Sondhi, Ann. Phys. (Amsterdam) 313, 497 (2004).

[53] G. Y. Cho and J. E. Moore, Ann. Phys. (Amsterdam) 326, 1515 (2011).

[54] C. W. von Keyserlingk, F. J. Burnell, and S. H. Simon, Phys. Rev. B 87, 045107 (2013).
[55] S. M. Kravec and J. McGreevy, Phys. Rev. Lett. 111, 161603 (2013).

[56] D. Gaiotto, A. Kapustin, N. Seiberg, and B. Willett, J. High Energy Phys. 02 (2015) 172.

[57] K. Hasebe, Nucl. Phys. B886, 952 (2014).

[58] M. Cirio, G. Palumbo, and J. K. Pachos, Phys. Rev. B 90, 085114 (2014).

[59] X. Chen, A. Tiwari, and S. Ryu, Phys. Rev. B 94, 045113 (2016).

[60] AtMa P. O. Chan, T. Kvorning, S. Ryu, and E. Fradkin, Phys. Rev. B 93, 155122 (2016).

[61] A. Cappelli, E. Randellini, and J. Sisti, J. High Energy Phys. 05 (2017) 135.

[62] M. K. Murray, J. Lond. Math. Soc. 54, 403 (1996).

[63] M. K. Murray, in The Many Facets of Geometry: A Tribute to Nigel Hitchin (Oxford University Press, Oxford, 2010).

[64] K. Waldorf, Surface Holonomy: Handbook of PseudoRiemannian Geometry and Supersymmetry, IRMA Lectures in Mathematics and Theoretical Physics (European Mathematical Society, 2006), p. 653.

[65] C. Saemann and R. J. Szabo, Rev. Math. Phys. 25, 1330005 (2013).

[66] M. Gell-Mann, Phys. Rev. 125, 1067 (1962).

[67] The hyperspherical coordinates are defined as $q_{x}=r \cos \theta_{1}, q_{y}=r \sin \theta_{1} \cos \theta_{2}, q_{z}=r \sin \theta_{1} \sin \theta_{2} \cos \varphi$, $q_{w}=r \sin \theta_{1} \sin \theta_{2} \sin \varphi$.

[68] T. Neupert, L. Santos, S. Ryu, C. Chamon, and C. Mudry, Phys. Rev. B 86, 035125 (2012).

[69] A. Celi, P. Massignan, J. Ruseckas, N. Goldman, I. B. Spielman, G. Juzeliunas, and M. Lewenstein, Phys. Rev. Lett. 112, 043001 (2014).

[70] H. M. Price, O. Zilberberg, T. Ozawa, I. Carusotto, and N. Goldman, Phys. Rev. Lett. 115, 195303 (2015).

[71] L. Asteria, D. T. Tran, T. Ozawa, M. Tarnowski, B. S. Rem, N. Fläschner, K. Sengstock, N. Goldman, and C. Weitenberg, arXiv:1805.11077. 